\title{
Structural, Optical, Electrochemical and Photovoltaic Studies of Spider Web like Silver Indium Diselenide Quantum dots Synthesized by Ligand Mediated Colloidal Sol-gel Approach
}

Tham Adhikari ${ }^{1}$, Dinesh Pathak ${ }^{2 *}$, Tomas Wagner ${ }^{2}$, Roman Jambor ${ }^{2}$, Uzma Jabeen ${ }^{1}$, Muhammad Aamir ${ }^{1}$, and Jean-Michel Nunzi ${ }^{*}$

${ }^{1}$ Department of Chemistry and Physics, Queen's University, Kingston, Ontario, Canada

2 Department of General and Inorganic Chemistry, University of Pardubice, Czech Republic

\section{dineshpathak80@gmail.com}

\section{nunzijm@queensu.ca}

\begin{abstract}
Silver indium diselenide quantum dots were successively synthesized by colloidal sol-gel method by chelating with organic ligand oleylamine (OLA). The particle size was studied by transmission electron microscopy (TEM) and the size was found about $10 \mathrm{~nm}$. X-ray diffraction (XRD) was used to study crystalline structure of the nanocrystals. The grain size and morphology were further studied by scanning electron microscopy (SEM) and atomic force microscopy (AFM). The elemental composition was studied by X-ray photon electron spectroscopy (XPS) and energy dispersive x-ray spectroscopy (EDAX). The capping property of OLA in nanocrystal was also demonstrated by Fourier Transform Infrared spectroscopy (FTIR). The band gap was calculated from both cyclic voltammetry and optical absorption and suggest quantum confinement. The solution processed bilayer thin film solar cells were fabricated with n-type Zinc oxide using doctor blading/spin coating method and their photovoltaic performance was studied. The best device sintered at $450{ }^{\circ} \mathrm{C}$ showed an efficiency $0.75 \%$ with current density of $4.54 \mathrm{mAcm}^{-2}$, open-circuit voltage $0.44 \mathrm{~V}$ and fill factor $39.4 \%$.
\end{abstract}

Key words: Nanocrystal, Quantum dots, ligand, oleylamine, solar cells, power conversion efficiency. 


\section{Introduction}

In the exploration of novel functional materials with improved optoelectronic properties, semiconductor nanocrystals are important materials for optical devices. ${ }^{1}$ Colloidal sol-gel method gives a large variety of compounds with an inorganic core and a corona of organic stabilizing ligands. ${ }^{2}$ Semiconductor nanocrystals, which are smaller in size, show unique optical and electrical properties. ${ }^{3}$ These properties are results of quantum confinement of an electron and hole so the semiconductor nanocrystals are called quantum dots. ${ }^{4}$ Band broadening of nanocrystals with crystal size has widely applicable in optical devices. ${ }^{5,6}$ Chalcopyrite is one common crystal structure of I-III- $\mathrm{VI}_{2}$ semiconductors with I and III ions ordering in the anion sub lattice sites. ${ }^{7}$ In recent years, the colloidal nanocrystals of the type I-III-VI 2 have attracted great attention in photovoltaic and non-linear optical devices due to their unique physical and chemical properties such as low toxicity and high absorption ${ }^{8}$ and the size and shape-tunable band gap. ${ }^{9-11}$ Silver indium diselenide $\left(\mathrm{AgInSe}_{2}, \mathrm{AIS}\right.$ ), a ternary semiconductor, has been widely used in a number of optoelectronic devices because of its low band gap (1.2-1.9 eV), high absorption coefficient $\left(\sim 10^{5} \mathrm{~cm}^{-1}\right)$ and ternary analogue of Cd Se. ${ }^{9}$ Despite the broad research on the ternary and quaternary chalcopyrite based thin film solar cells, monocrystalline silicon solar cells are still dominated in industries because of several factors such as relatively high manufacturing cost in their vacuum deposition process, the difficulty in achieving controllable and uniform composition over large area. ${ }^{12-14}$ Cadmium based quantum dots have been used as light absorber since past decade due to their optical properties. However, their applications are becoming more and more limited because of their toxicity. ${ }^{5,6}$

Several techniques have been reported to synthesize $\mathrm{AgInSe}_{2}$ macro and nanocrystals that require either high vacuum and high temperature. ${ }^{15,16}$. The problem of the vacuum based technique is its high manufacturing cost and low stoichiometric composition of Se due to the high volatility of Se-containing compounds. So, it was realized to develop solution based techniques to synthesize ternary and quaternary nanocrystals. Al-Agel \& Ghamdi et al developed a new strategy to synthesize a $\mathrm{AgInSe}_{2}$ thin film by using spin coating method. They also studied the influence of annealing temperature on composition and the crystal structure. ${ }^{17}$ Recently, the colloidal solution approaches are more effective and powerful for synthesizing nanocrystals with designed structure and shapes. ${ }^{3,18}$ 
In this communication, we report a simple and relatively green colloidal sol-gel method for synthesizing ternary $\mathrm{AgInSe}{ }_{2}$ nanocrystals by using oleylamine as a chelating ligand/organic solvent. It has been reported that amine can lower the thermal decomposition temperature of the precursor and facilitate the formation of nanoparticles at lower temperature. ${ }^{9}$ In addition, oleylamine (OAm) and dodecanethiol (DT) have been widely used as surfactants for synthesizing various nanoparticles and are reported as reagents that promote the anisotropic growth of nanoparticles. ${ }^{19}$ Furthermore, they have unique advantages such as high boiling point, low cost, tendency to form metal-OAm complexes at intermediate temperature so that it can controllably decomposed to produce nanoparticles. OAm can form complex compounds with metal ions of the corresponding precursor leading to metastable compounds that can act as secondary precursor and thus be decomposed in a controlled way to yield nanoparticle. ${ }^{20}$

Very few works have been reported for solution processed thin film devices using colloidal sol-gel nanocrystals. Our group already reported ternary blend polymer based bulkheterojunction solar cells by incorporating AIS nanocrystal prepared by ball mill method. ${ }^{21-23}$ As far our knowledge, we are first to report solution processed bilayer device fabricated using AIS nanocrystal prepared by colloidal sol-gel method. The bilayer thin films devices ITO/AIS/ZnO/Al was fabricated on pre-patterned Indium oxide tin oxide deposited sequentially by doctor blading and spin coating followed by vacuum deposition of aluminum. All the devices sintered at different temperatures showed some photovoltaic performance with highest power conversion efficiency $0.75 \%$. This work will be promising to fabricate easily processable solution processed devices using sol-gel synthesized ternary quantum dots which are necessary for flexible devices.

\section{Materials and Methods}

\section{Materials}

Selenium powder (Se, 99.99\%) and oleylamine (OAm, 70\%) were purchased from SigmaAldrich. Silver acetate (99.8\%) and Indium acetate (99.5\%) were purchased from Alfa Aesar. All the chemicals were used as received without purification. $\mathrm{ZnO}$ nanopowder (size $10 \mathrm{~nm}$ ) were purchased from Alfa Aesar. The patterned Indium tin oxide (ITO) glasses (film thickness about $135 \pm 15 \mathrm{~nm}$, and sheet resistance is $15 \Omega \mathrm{sq}^{-2}$ ) used as substrates were purchased from Luminescence Technology Corporation. 


\section{Synthesis of AgInSe 2 Quantum dots}

A solution of silver acetate $(2 \mathrm{mmole}, 0.33 \mathrm{~g})$ and Indium acetate $(2 \mathrm{mmole}, 0.58 \mathrm{~g})$ in $30 \mathrm{ml}$ of oleylamine was heated at $180^{\circ} \mathrm{C}$ under a nitrogen atmosphere with continuous magnetic stirring for 30 minutes resulting a transparent yellow solution as shown in Figure S1. Next, Selenium (1.58 g, $4 \mathrm{mmol}$ ) was dissolved in $15 \mathrm{~mL}$ oleylamine by heating to $120{ }^{\circ} \mathrm{C}$ with constant stirring in vacuum. The Se solution was injected hot into the original solution resulting immediately black brown turbid precipitate as presented in Figure S1 and the temperature was gradually increased up to $200{ }^{\circ} \mathrm{C}$ in nitrogen atmosphere under magnetic stirring. The solution was kept at that temperature for $1 \mathrm{hr}$. Finally, the product was cooled rapidly to grow $\mathrm{AgInSe}{ }_{2}$ nanocrystals.

The AgInSe2 nanocrystals were separated from resulting solution by centrifugation three times with methanol and then with toluene at $4000 \mathrm{rpm}$. The nanocrystals were washed with methanol and dried in an oven at $60{ }^{\circ} \mathrm{C}$ for overnight. The dried $\mathrm{AgInSe}_{2}$ nanocrystals were used for characterization and device fabrication.

\section{Characterization}

UV-visible absorption spectra were acquired using an Olid ${ }^{\circledR}$ HP8452 Diode Array Spectrometer. Luminescence was measured with a USB2000-Ocean Optics spectrometer. XRD were obtained using an Xpert Pro Philips powder X-ray diffractometer to check phase purity and crystalline nature nanoparticles. Field emission gun scanning electron microscope (FEI Quanta 650 FEG) supplemented with EDAX (Bruker XFlash detector 1.9) was used for knowing the morphology, size and composition of nanocrystals. A transmission electron microscope (Hitachi H-7000, 75 W) was used knowing morphology of nanocrystals. FTIR spectra were obtained with a Nicolet 6700 in the ATR mode. The morphology of nanoparticles and films were characterized using an Ambios multimode Atomic Force Microscope (AFM) in tapping mode with $300 \mathrm{KHz}$ resonant frequency cantilever. The XPS spectra were measured on a Microlab 310-F spectrometer equipped with an XR-4 twin anode $(\mathrm{Al} / \mathrm{Mg})$. The samples were mounted on a stubtype stainless steel holder using double-sided adhesive $\mathrm{Cu}$ tape and kept under high vacuum $\left(10^{-8}\right.$ mbar) overnight inside the preparation chamber before they were transferred into the analysis chamber $\left(10^{-9} \mathrm{mbar}\right)$ of the spectrometer. The cyclic voltammograms were recorded in the Keithly system connected with potentiostat consisting of three electrodes: working electrode (glassy carbon-carbon electrode), reference electrode $(\mathrm{Ag} / \mathrm{AgCl}$ (in $3 \mathrm{M} \mathrm{NaCl}$ ) and counter or 
auxiliary electrode ( $\mathrm{Pt}$ electrode) in $0.1 \mathrm{M}$ tetra butyl ammonium phosphorus Hexafluoride in acetonitrile. Current density-voltage (J-V) measurements were carried out using a Keithley 4200SCS in the dark and under illumination. The current density-voltage (J-V) photovoltaic measurements of solar cells were carried out by employing an AM 1.5 solar simulator equipped with a xenon lamp. The power of the simulated light was calibrated to $100 \mathrm{~W} / \mathrm{m}^{2}$ by using a reference Si photo-diode.

\section{Results and Discussions}

\section{Structural and Optical Properties}

The typical high resultion transmission electron microscopy image of the resulting $\mathrm{AgInSe} \mathrm{I}_{2}$ dots is shown in Figure 1. The nanoparticles exhibit clusters of varying shape with surfactant at high concentration as shown in Figure 1(a). However, it shows the distinct nanoparticles after dilution with methanol shown in Figure 1(b). The average size of nanoparticles is about $10 \pm 2 \mathrm{~nm}$.
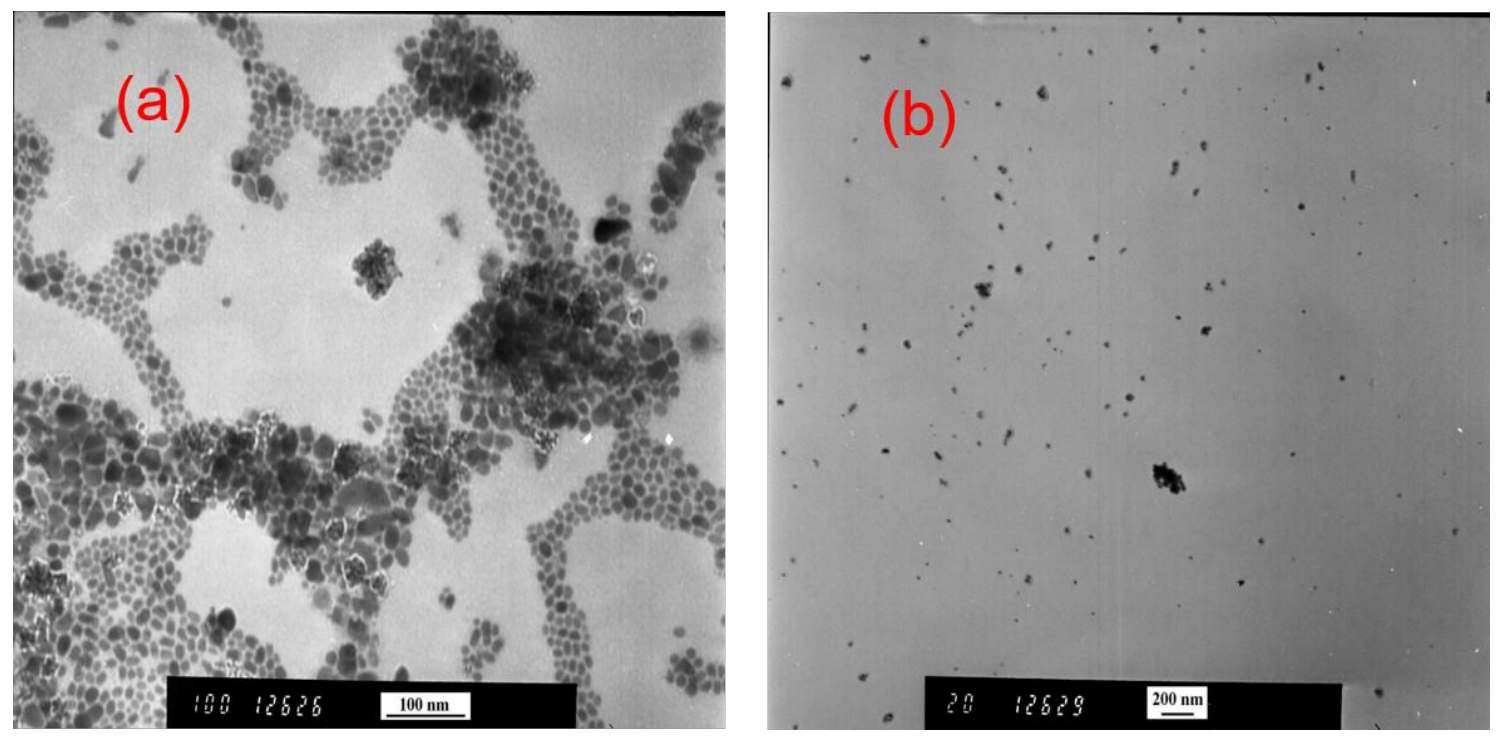

Figure 1 HRTEM images for AgInSe2 nanoparticle dispersed in methanol (a) in high and (b) in low concentration.

Scanning Electron Microscope (SEM) was used for studying the surface morphology and the micro structural features of $\mathrm{AgInSe}_{2}$ nanocrystals. 

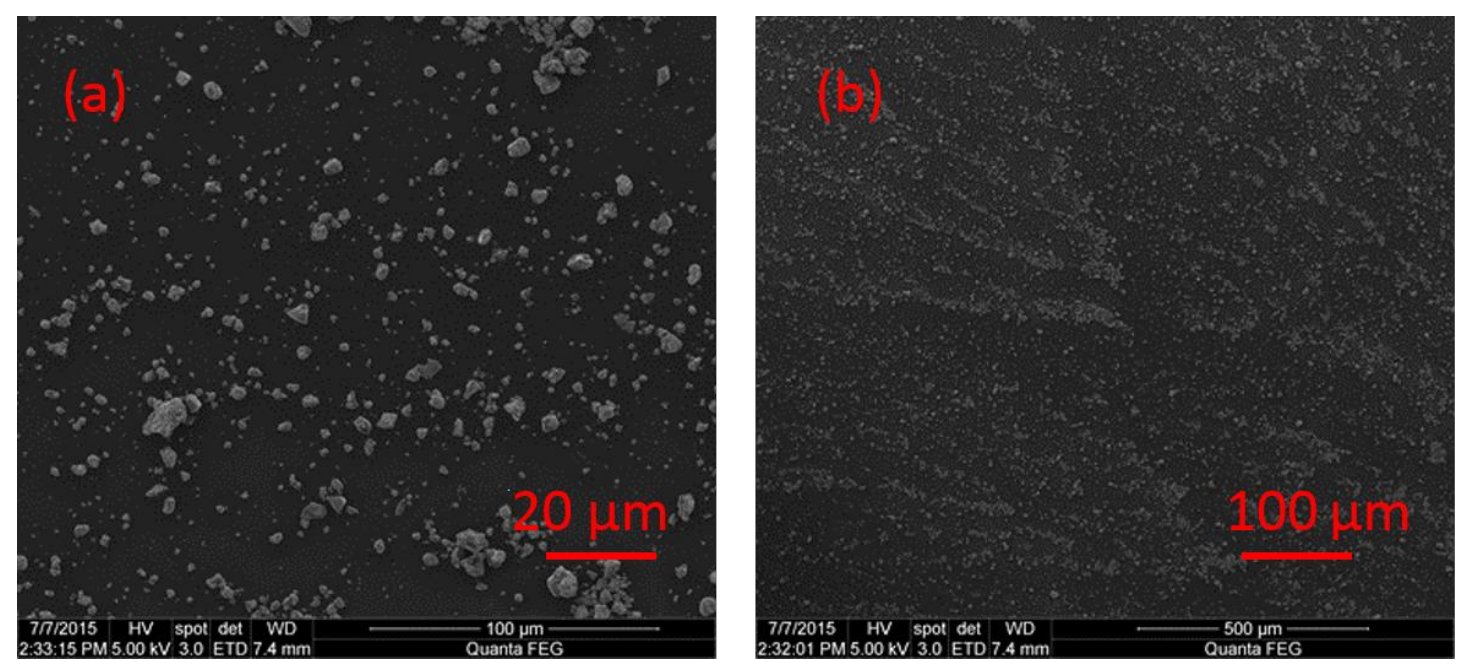

Figure 2 Scanning electron micrograph for $\mathrm{Ag} \mathrm{InSe}_{2}$ nanocrystals (a) in $100 \mu \mathrm{m}$ (b) in $500 \mu \mathrm{m}$.

Scanning electron microscopy (SEM) was used to study the surface morphology and the micro structural features of the thin film. SEM image was obtained for AgInSe2 thin film on glass substrate and shown in Figure 2. It shows a compact structure with densely packed, spherical and well defined grain with similar size nanocrystals.

Energy Dispersive X-ray Spectrometer (EDS) was carried out to determine the compositional analyses of $\mathrm{AgInSe} \mathrm{I}_{2}$ nanoparticles. EDS elemental map for nanoparticle is shown in Figure S2. The three elements Ag, In, and Se are identified in all the nanocrystals, and no apparent element separation or aggregation was observed, indicating that all three elements are equally distributed among the nanocrystals. The spectrum in Figure S2 shows the presence of Ag, In and Se peaks confirming the formation of $\mathrm{AgInSe}_{2}$. However, an additional peak of oxygen is also appeared. This may be due to $\mathrm{In}_{2} \mathrm{O}_{3}$ produced by the thermal decomposition of Indium acetate in OAm under inert condition as shown in reaction. The average atomic percentage ratio of $\mathrm{Ag}$ : In: $\mathrm{Se}$ is shown in Table S1.

In addition, the chemical analysis of the $\mathrm{AgInSe}{ }_{2}$ nanoparticles was further analyzed by X-ray photoelectron spectra (XPS). Figure $3 \mathrm{a}$ shows the binding energies of $\mathrm{Ag} 3 \mathrm{~d}_{3 / 2}$ and $\mathrm{Ag} 3 \mathrm{~d}_{5 / 2}$ for $\mathrm{AgInSe}_{2}$ located at $375.8 \mathrm{eV}$ and $368.8 \mathrm{eV}$ with a peak separation of $7.0 \mathrm{eV}$. Figure $3 \mathrm{~b}$ shows the In $3 \mathrm{~d}$ peaks located at $453.4 \mathrm{eV}$ and $445.1 \mathrm{eV}$ with a peak separation of $8.2 \mathrm{eV}$. Figure $4 \mathrm{c}$ shows the Se $3 \mathrm{~d}$ peaks located at $55.3 \mathrm{eV}$. The results are all in agreement with the results reported in the literatures for $\mathrm{AgInSe}_{2}$ nanocrystals, suggesting that the valence states of the sample are $\mathrm{Ag}^{+}$, 
$\mathrm{In}^{3+}$, and $\mathrm{Se}^{2-} \cdot 39$
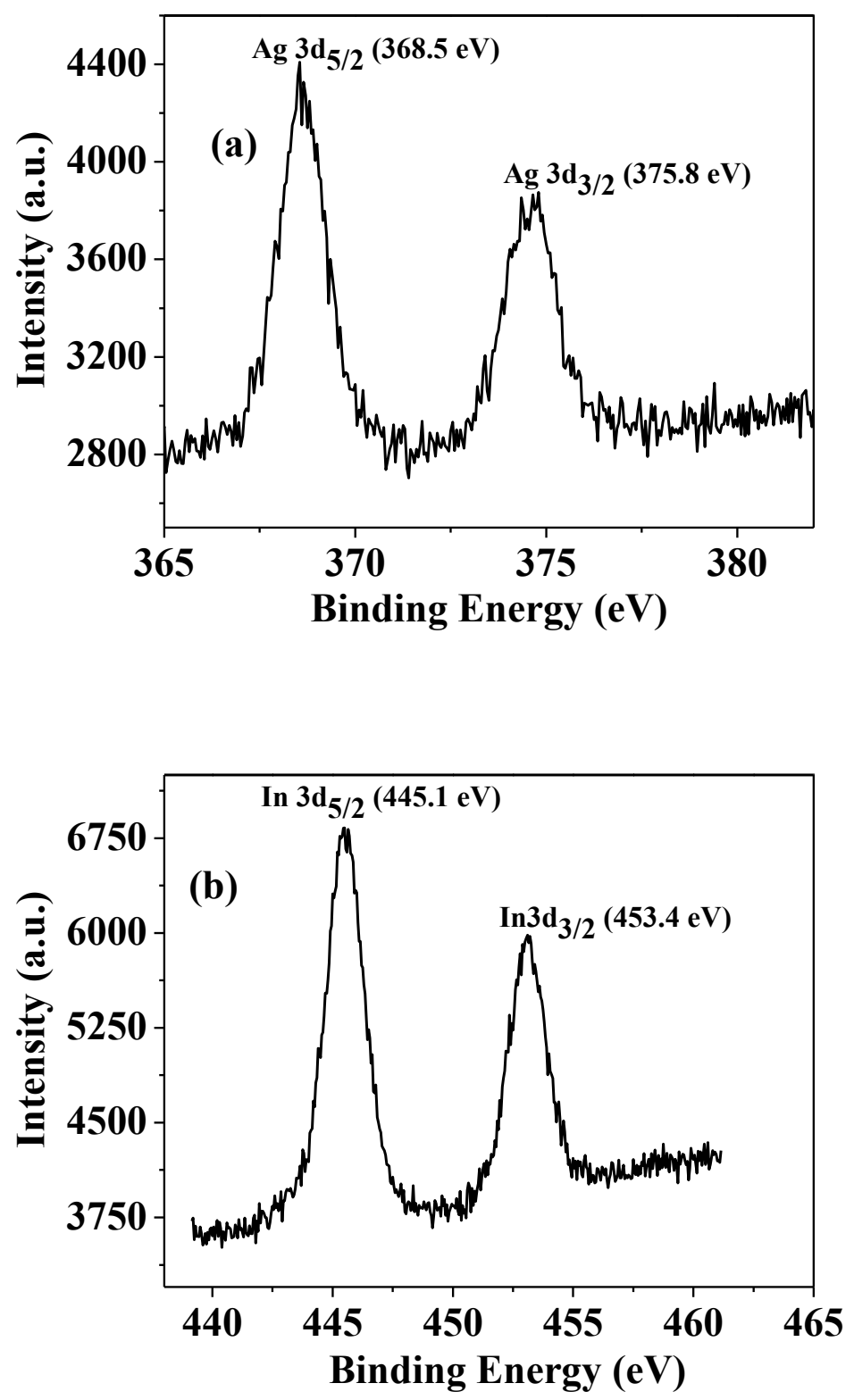


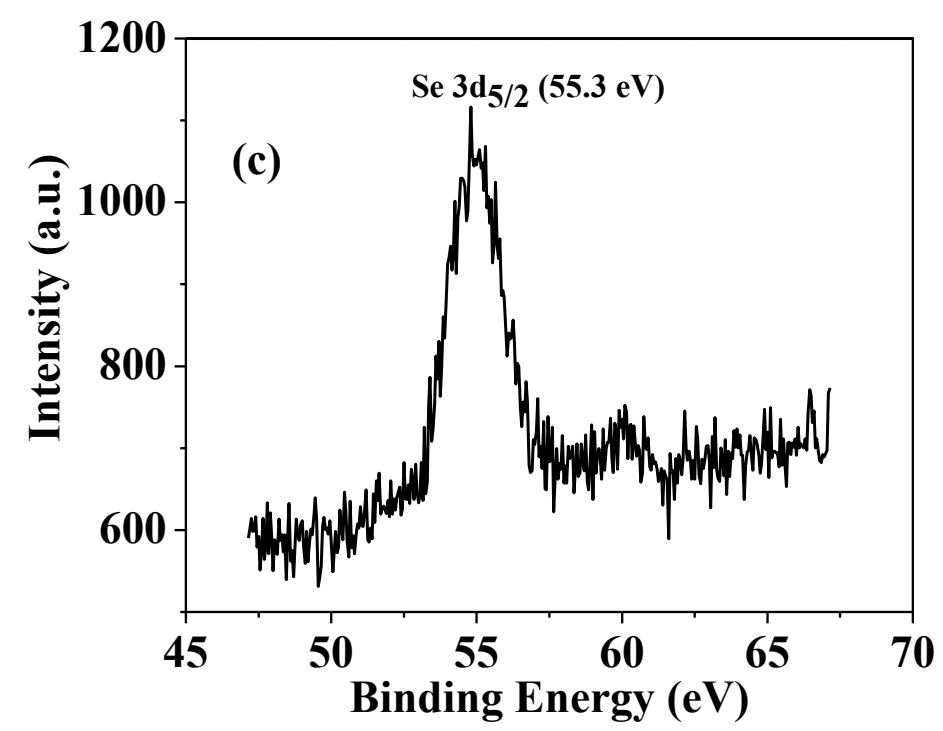

Figure 3 High resolution XPS spectra for (a) Ag3d (b) In 3d and (c) Se 3d.

X-ray measurements were performed using a Xpert Pro Phillips powder x-ray diffractometer with Cobalt x-ray tube $\left(\Lambda=1.78 \mathrm{~A}^{0}\right)$, Iron filter, graphite crystal monochromator, proportional counter detector, $0.5^{0}$ divergence slit and $0.25 \mathrm{~mm}$ receiving slit. The working conditions were 40 $\mathrm{kV}$ and $45 \mathrm{~mA}$ for the X-ray tube, with a scan speed of $0.05^{\circ}$ and $60 \mathrm{~s}$ of measuring time per step. The X-ray diffraction pattern is shown in Figure 4. The pattern shows the deflection peaks at reflection planes (112), (220) and (312), the chalcopyrite structure of AgInSe2 with lattice parameters as reported in the literature. ${ }^{17}$ The average diameter of crystal domains was calculated from the full width at half maximum (FWHM) by using Scherrer diffraction formula $\mathrm{D}=\mathrm{k} K / \beta \operatorname{Cos} \Theta$ where $\mathrm{k}$ is constant related to crystallite shape (normally 0.9 ), $\Lambda=0.178 \mathrm{~nm}$ is the $\mathrm{X}$-ray wavelength, $\Theta$ is the diffraction angle of the most intense peak at $2 \Theta=25^{\circ}$, and $\beta$ is diffraction angular width at the half maximum of this (112) plane. It was found that average crystallite size for the nanocrystal was about $12.4 \mathrm{~nm}$ which corresponds well with the particle size calculated from HRTEM. The peaks are quiet broad as desired for fine nanoparticles. 


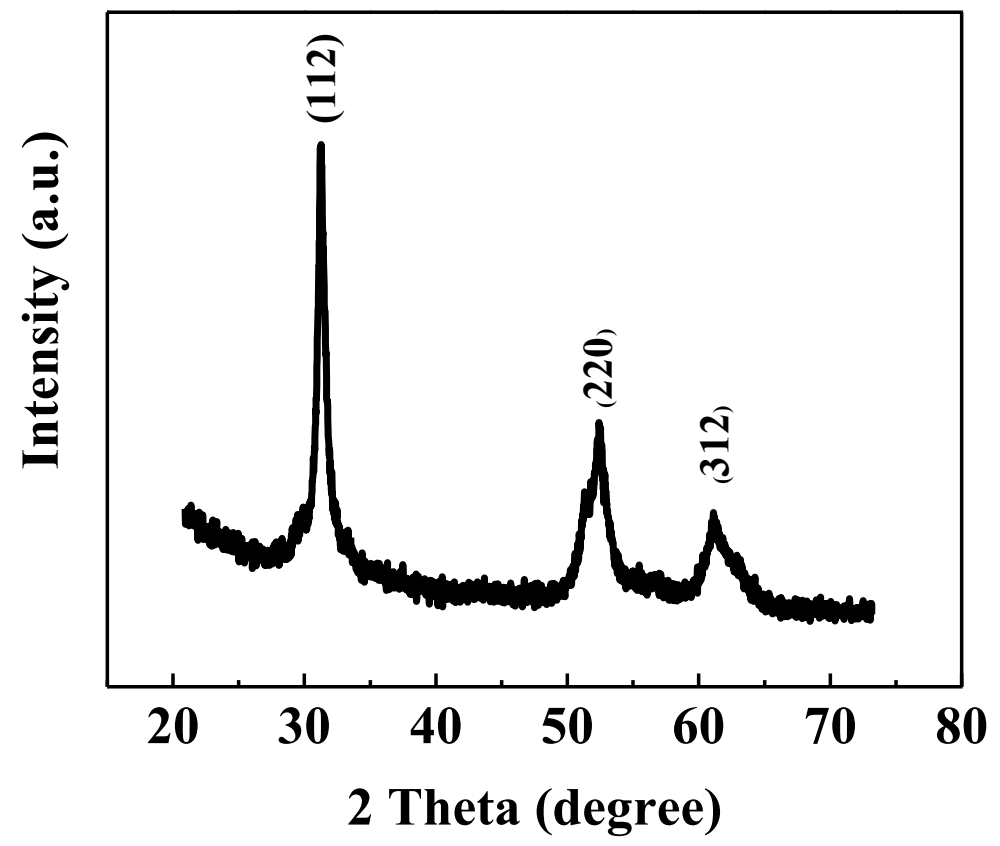

Figure 4 X-ray diffraction pattern for AgInSe2 nanocrystals.

Surface topographic image of AIS nanocrystal on $\mathrm{ZnO}$ on cleaned glass substrate was measured by taping mode atomic force microscope (AFM). The 2D and 3D topographic image are shown in Figure S3. It shows embeded nanoparticles with smooth morphology. The root mean square roughness was $22.2 \mathrm{~nm}$.

UV -Visible absorption spectrum for AIS nanocrystals is shown in Figure 5. It reveals an absorption onset at around $800 \mathrm{~nm}$ and a steep increase of the absorption towards lower wavelength. 


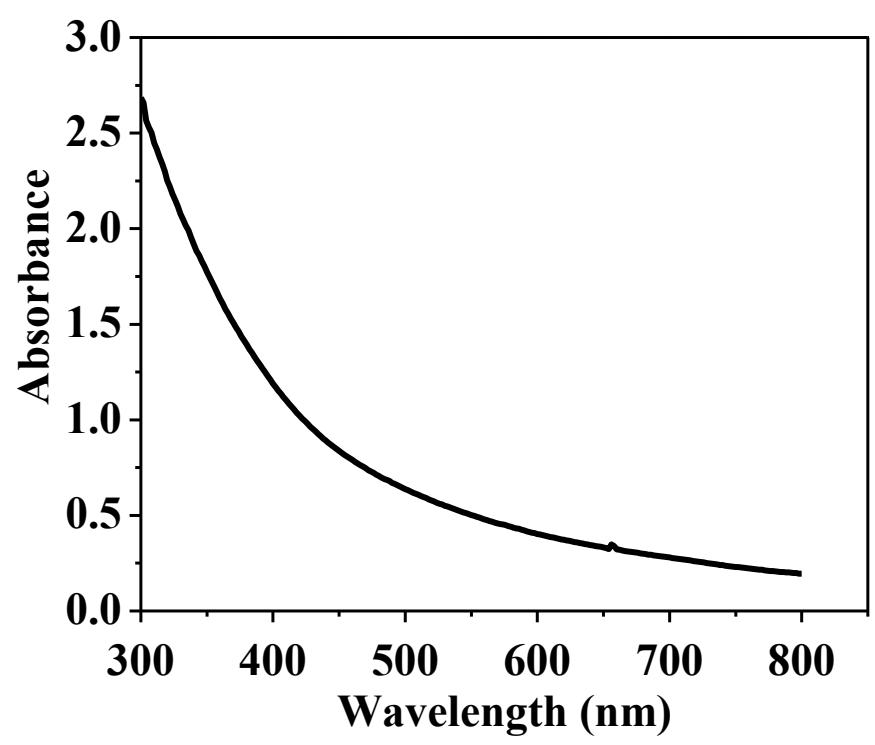

Figure 5 UV-Visible Spectra for AIS nanoparticle.

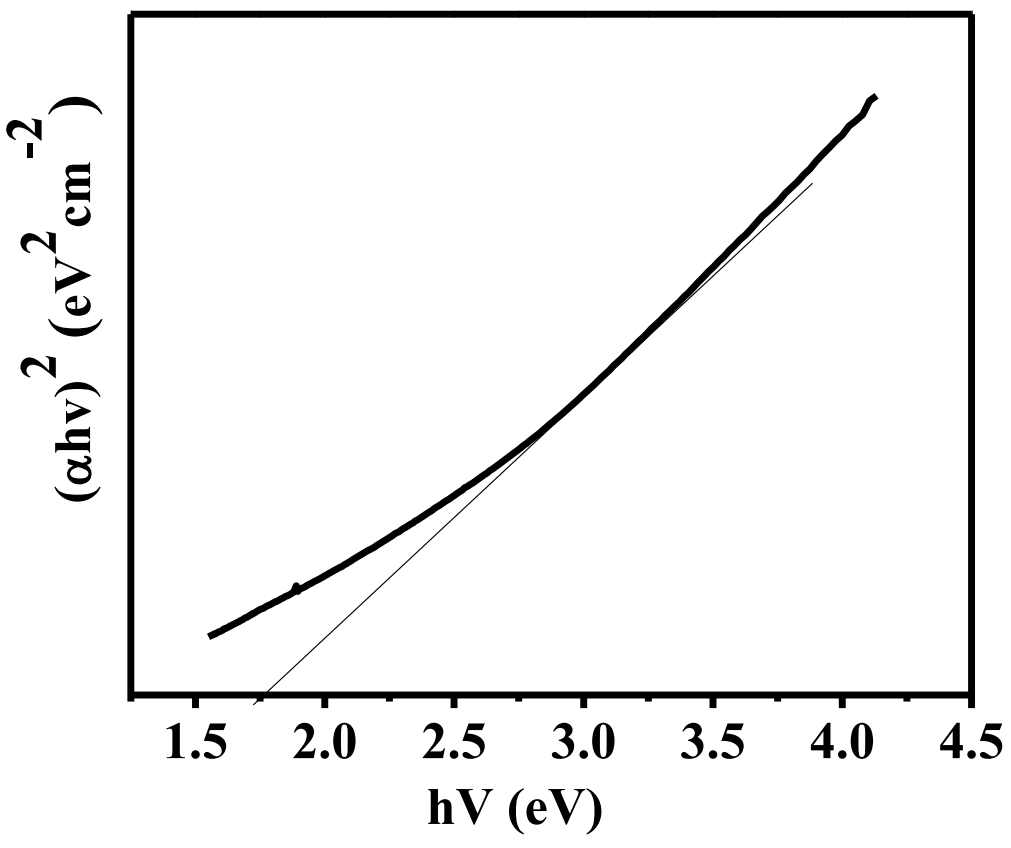

Figure 6 Optical Band gap for AIS nanoparticle.

The optical band gap is calculated using the following equation: $\alpha h \mathrm{~V}=\left(h \mathrm{~V}-E_{g}\right)^{\mathrm{n}}$. In this equation, $h \mathrm{~V}$ is the photon energy, $\alpha$ is the absorption coefficient of the thin film, $E g$ is the optical band gap, and $A$ is the bandwidth coefficient, which is a constant value. For the direct 
band gap semiconductor materials $n$ is $1 / 2$. The optical band gap is determined by extrapolating straight line of the plot $(\alpha h v)^{2}$ versus hv as shown in Figure 6. The optical band gap was found to be $1.75 \mathrm{eV}$. A blue shift is observed increasing the band gap which suggest quantum confinement as size of the particles appears to lie in bohr's exciton regime for $\mathrm{AgInSe}_{2}$. The observed band gap corresponds well with the reported value and theoretical predictions for quantum confinement as reported in the literature. ${ }^{24,25}$

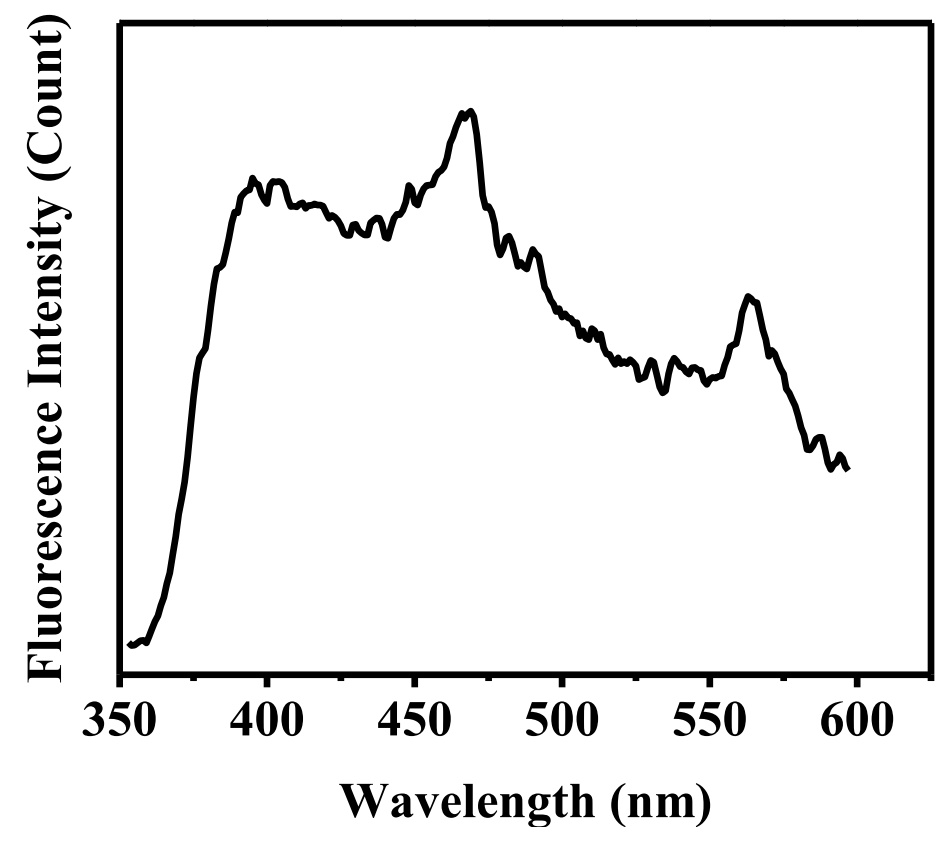

Figure 7 Fluorescence spectra for $\mathrm{AgInSe} \mathrm{e}_{2}$ nanocrystal.

The fluorescence emission spectrum of AIS nanoparticles obtained by exciting the nanoparticles at $320 \mathrm{~nm}$ is shown in Figure 6. The fluorescence emission spectrum of the AIS nanoparticles was deconvoluted into three bands, which are centered at 419 and $458 \mathrm{~nm}$ and $568 \mathrm{~nm}$ respectively. Further, It shows a broad emission extending up to $600 \mathrm{~nm}$.

\section{Electrochemical properties for Nanocrystals}

The cyclic voltammograms were recorded in the Keithly system connected with potentiostat consisting of three electrodes: working electrode (glassy carbon-carbon electrode), reference electrode $(\mathrm{Ag} / \mathrm{AgCl}$ (in $3 \mathrm{M} \mathrm{NaCl})$ and counter or auxiliary electrode (Pt electrode) in $0.1 \mathrm{M}$ tetra butyl ammonium phosphorus Hexafluoride in acetonitrile at scan rate $100 \mathrm{mVs}-1$ with bubbling of N2 gas. All nanocrystals samples were purified and dissolved in toluene. 1-2 mg nanocrystals 
deposited directly on glassy carbon electrode. The electrolyte solutions were thoroughly degassed with nitrogen for $30 \mathrm{~min}$; and the scan rate was set at $50 \mathrm{mV} / \mathrm{s}$.

The cyclic voltamogram is shown Figure S5 (in supporting information). From CV in Figure S5, the band-edge positions (i.e., the highest occupied molecular orbital (HOMO) and lowest unoccupied molecular orbital (LUMO) energy position) can be determined. Oxidation potential was correlated directly with the ionization potential (Ip) and the reduction potential with the electron affinity $(\mathrm{Ea})$, the band edge positions of electroactive materials can be calculated. The HOMO and LUMO energy levels can be derived from the onset oxidation potential (Eox) and onset reduction potential (Ered), respectively according to equation reported in the literature. The details for calculation shown in supporting information in Table S2. The calculated LUMO and HOMO levels are -4.0 and $-5.9 \mathrm{eV}$ respectively and gap is $1.70 \mathrm{eV}$ corresponds to optical gap $(1.75 \mathrm{eV})$.

\section{Device Fabrication}

Solar cells of ITO/AIS/Zno/Al structures were fabricated on transparent conductive indium tin oxide (ITO) coated glass substrates. Pre-patterned indium tin oxide (ITO) coated glass slides were cleaned by ultrasonication for 10 minutes in detergent water, distilled water, acetone and isopropyl alcohol sequentially. The substrates were dried with air blower and further dried on hot plate at $100{ }^{\circ} \mathrm{C}$ for 20 minutes. The dried substrates were further subjected to oxygen plasma cleaning with for 15 minutes. The AIS suspension in 2-propanol was doctor bladed on cleaned ITO-substrate and sintered at 350, 400, 450 and $500{ }^{\circ} \mathrm{C}$ respectively. $\mathrm{ZnO}$ nanoparticles $(0.16 \mathrm{~g} / \mathrm{mL}$ in 2-propanol) was spin-coated on cleaned ITO substrate at a rate of $1000 \mathrm{rpm}$ in air. The substrates coated with $\mathrm{ZnO}$ were baked at $200{ }^{\circ} \mathrm{C}$ for $1 \mathrm{~h}$. The thickness of $\mathrm{ZnO}$ was approximately 45-50 $\mathrm{nm}$ as measured with a Sloan Dektak II profilometer. The top and bottom electrodes of the devices were cleaned with a cotton stick to make contacts. Finally, $100 \mathrm{~nm}$ of Al were deposited with deposition rates $0.4 \AA / \mathrm{s}$ and $1.5 \AA / \mathrm{s}$ respectively on all the devices using

a physical vapour deposition system (PVD) under high vacuum at a pressure of $6 \times 10^{-6}$ torr. The active area of the device was $0.06 \mathrm{~cm}^{2}$. The thin film device scheme is shown in Figure 8 . 


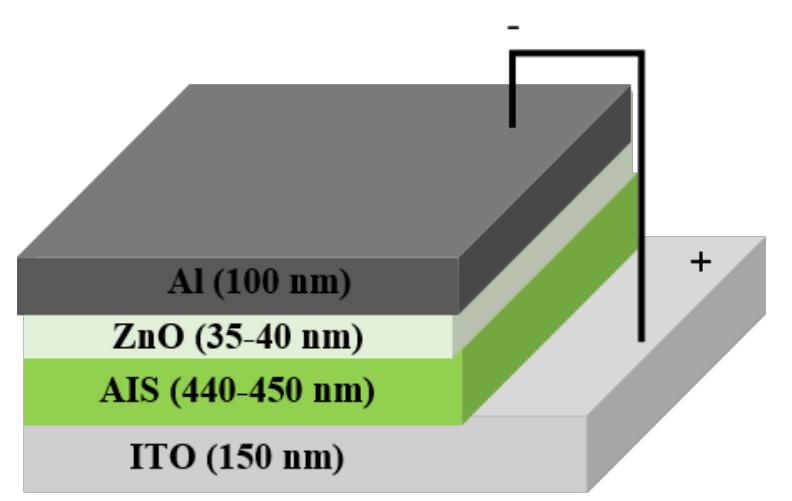

Figure 8 Thin film device structure for ITO/AIS/ZnO/Al.

\section{Device Characterization}

Photovoltaic measurements were performed in ambient condition using light from a Xenon source calibrated with an AM 1.5 solar simulator to reproduce an incident light intensity of 100 $\mathrm{mWcm}^{-2}$. The measurements were carried out at ambient atmosphere and the I-V characteristics of PV device ITO/AIS/ZnO/Al in dark and light are shown in Figure 8. The power conversion efficiency (PCE) was calculated from the value of open-circuit voltage $\left(\mathrm{V}_{\mathrm{oc}}\right)$, short-circuit current density $\left(\mathrm{J}_{\mathrm{sc}}\right)$ and fill factor $(\mathrm{FF})$ with the following equation: $\mathrm{PCE}=\mathrm{P}_{\text {out }} / \mathrm{P}_{\text {in }}=$ $J_{\mathrm{sc}} \times V_{\mathrm{oc}} \times F F / P_{\text {in }}$, where $P_{\text {in }}$ represents the power of incident light $\left(\mathrm{mW} / \mathrm{cm}^{2}\right)$. The fill factor was calculated from the values of $\mathrm{V}_{\mathrm{oc}}, \mathrm{J}_{\mathrm{sc}}$, and maximum power $\left(\mathrm{P}_{\max }\right)$ according to the following equation: $\mathrm{FF}=\mathrm{P}_{\max } / \mathrm{J}_{\mathrm{sc}} \times \mathrm{V}_{\mathrm{oc}}=\mathrm{J}_{\max } \times \mathrm{V}_{\max } / \mathrm{J}_{\mathrm{sc}} \times \mathrm{V}_{\mathrm{oc}}$, where $\mathrm{J}_{\max }$ and $\mathrm{V}_{\max }$ are the current density and voltage at maximum power respectively. The photovoltaic parameters of devices device ITO/AIS/ZnO/Al are reported in Table 1.

The dark IV in Figure 8 a shows the devices sintered at $450{ }^{\circ} \mathrm{C}$ shows significantly lower leakage current compared to one processed at other temperatures and it shows better photovoltaic performance. This is attributed to the better crystal quality. It is reported that higher concentration of defects at the growth interface resulted in relatively larger leakage current. ${ }^{26}$ Figure $8 \mathrm{~b}$ shows the PV performance in light. All the devices sintered at various temperature $\left(350-500{ }^{\circ} \mathrm{C}\right)$ show some photovoltaic performance efficiencies ranging from $0.19-0.75 \%$ efficiency. Furthermore, it is seen that the efficiency increases as the sintering temperature increases and after optimum temperature $450{ }^{\circ} \mathrm{C}$ it starts to decreases. The better performance at higher sintering temperature is attributed to higher conductivity of AIS. However, the lower 
efficiency is observed surprisingly at $500{ }^{\circ} \mathrm{C}$. It could be due to formation of pin holes/ undesired voids between the sintered nanocrystals at higher temperature. ${ }^{27,28}$ The best device shows current density $4.50 \mathrm{mAcm}-2$, open-circuit voltage $0.42 \mathrm{~V}$ and fill factor $39.4 \%$. The results reported here is the highest efficiency for the solution processed bilayer thin film solar cells with silver indium diselenide as light absorber.
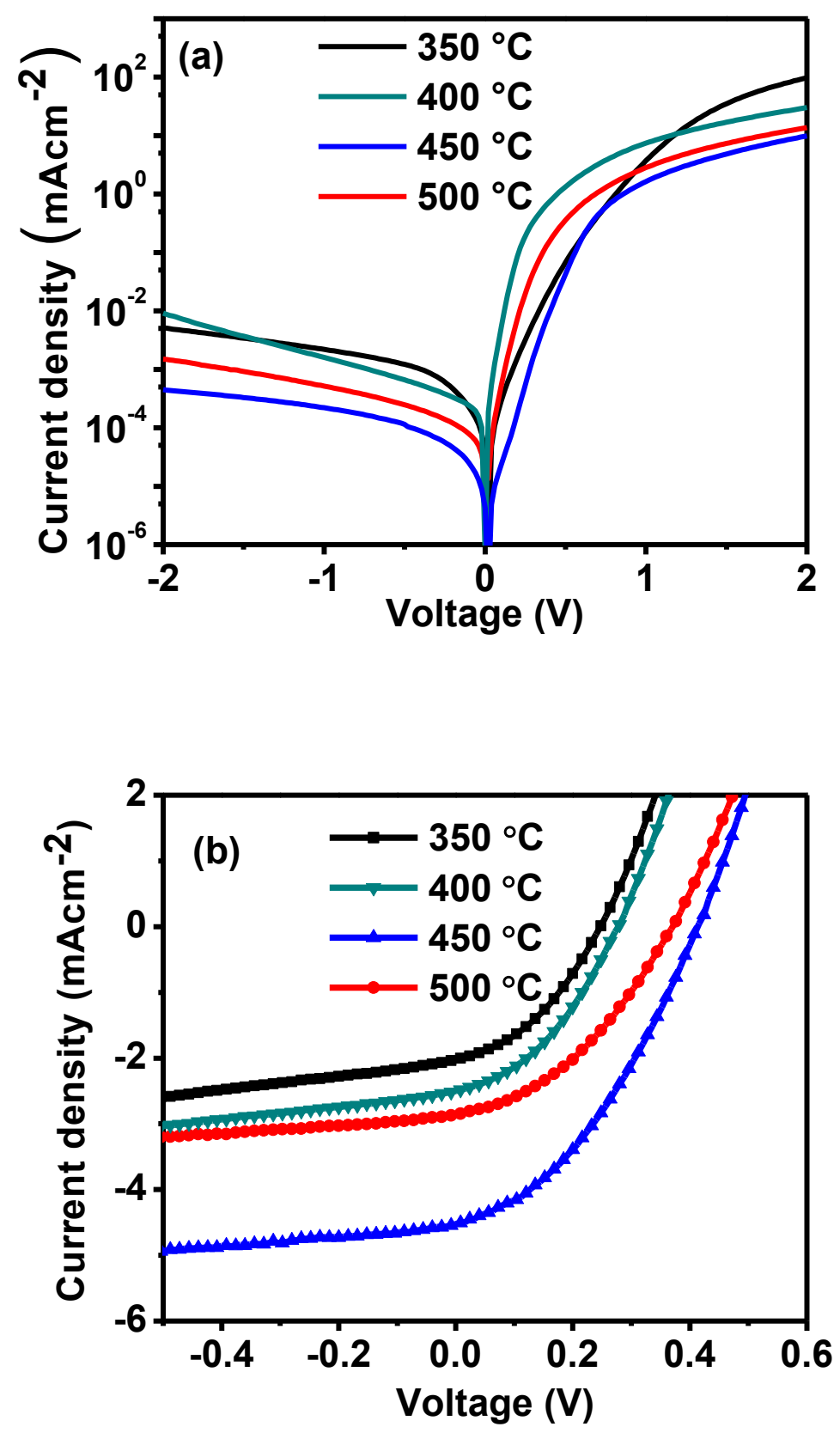
Figure 8 Current density-voltage (J-V) curves for ITO/AIS/ZnO/Al, (a) in Dark \& (b) in light of $100 \mathrm{mWcm}^{-2}$.

Table 1 Photovoltaic parameters for the devices ITO/AIS/ZnO/Al.

\begin{tabular}{|l|l|l|l|l|}
\hline Sintering Temp $\left({ }^{\circ} \mathrm{C}\right)$ & Jsc $\left._{(\mathbf{m A c m}}{ }^{-2}\right)$ & Voc (V) & FF (\%) & PCE (\%) \\
\hline 350 & $2.02 \pm 0.19$ & $0.25 \pm 0.06$ & $36.2 \pm 0.13$ & $0.19 \pm 0.10$ \\
\hline 400 & $2.50 \pm 0.17$ & $0.28 \pm 0.02$ & $37.3 \pm 0.15$ & $0.27 \pm 0.13$ \\
\hline 450 & $4.52 \pm 0.02$ & $0.42 \pm 0.03$ & $39.4 \pm 0.10$ & $0.75 \pm 0.09$ \\
\hline 500 & $2.86 \pm 0.15$ & $0.37 \pm 0.01$ & $38.5 \pm 0.05$ & $0.41 \pm 0.11$ \\
\hline
\end{tabular}

\section{Conclusion}

To summarize, we have synthesized $\mathrm{AgInSe}_{2}$ quantum dots in an organic chelating agent as reaction medium at low temperature processing condition and fabricated thin film devices with n-type $\mathrm{ZnO}$. All the devices sintered at various temperature $\left(350-500{ }^{\circ} \mathrm{C}\right)$ show some photovoltaic performance efficiencies ranging from $0.19-0.75 \%$ efficiency. The best device shows current density $4.50 \mathrm{mAcm}-2$, open-circuit voltage $0.42 \mathrm{~V}$ and fill factor $39.4 \%$. The results reported here is the highest efficiency for the solution processed bilayer thin film solar cells with silver indium diselenide as light absorber synthesized by colloidal sol-gel approach. This work will be promising to fabricate solution processed devices using sol-gel quantum dots which is necessary for flexible devices.

\section{References}

1. Buchmaier, C.; Rath, T.; Pirolt, F.; Knall, A.-C.; Kaschnitz, P.; Glatter, O.; Wewerka, K.; Hofer, F.; Kunert, B.; Krenn, K.; Trimmel, G., Room temperature synthesis of CuInS2 nanocrystals. $R S C A d v$. 2016, 6, 106120-106129.

2. Aldakov, D.; Lefrançois, A.; Reiss, P., Ternary and quaternary metal chalcogenide nanocrystals: synthesis, properties and applications. Journal of Materials Chemistry C 2013, 1 , 3756-3776. 
3. Nose, K.; Omata, T.; Otsuka-Yao-Matsuo, S., Colloidal synthesis of ternary copper indium diselenide quantum dots and their optical properties. The Journal of Physical Chemistry C 2009, 113, 3455-3460.

4. $\quad$ Bai, T.; Li, C.; Li, F.; Zhao, L.; Wang, Z.; Huang, H.; Chen, C.; Han, Y.; Shi, Z.; Feng, S., A simple solution-phase approach to synthesize high quality ternary AgInSe2 and band gap tunable quaternary $\mathrm{AgIn}(\mathrm{S} 1-\mathrm{xSex}) 2$ nanocrystals. Nanoscale 2014, 6, 6782-9.

5. Carey, G. H.; Abdelhady, A. L.; Ning, Z.; Thon, S. M.; Bakr, O. M.; Sargent, E. H., Colloidal Quantum Dot Solar Cells. Chemical reviews 2015, 115, 12732-63.

6. Du, J.; Du, Z.; Hu, J. S.; Pan, Z.; Shen, Q.; Sun, J.; Long, D.; Dong, H.; Sun, L.; Zhong, X.; Wan, L. J., Zn-Cu-In-Se Quantum Dot Solar Cells with a Certified Power Conversion Efficiency of 11.6\%. Journal of the American Chemical Society 2016, 138, 4201-9.

7. Bucherl, C. N.; Oleson, K. R.; Hillhouse, H. W., Thin film solar cells from sintered nanocrystals. Current Opinion in Chemical Engineering 2013, 2, 168-177.

8. Wu, L.; Chen, S.-Y.; Fan, F.-J.; Zhuang, T.-T.; Dai, C.-M.; Yu, S.-H., Polytypic Nanocrystals of $\mathrm{Cu}$-Based Ternary Chalcogenides: Colloidal Synthesis and Photoelectrochemical Properties. Journal of the American Chemical Society 2016, 138, 5576-5584.

9. Ng, M. T.; Boothroyd, C. B.; Vittal, J. J., One-pot synthesis of new-phase AgInSe2 nanorods. Journal of the American Chemical Society 2006, 128, 7118-7119.

10. Sargent, E. H., Colloidal quantum dot solar cells. Nature Photonics 2012, 6, 133-135.

11. Suryawanshi, M. P.; Ghorpade, U. V.; Shin, S. W.; Pawar, S. A.; Kim, I. Y.; Hong, C. W.; Wu, M.; Patil, P. S.; Moholkar, A. V.; Kim, J. H., A Simple Aqueous Precursor Solution Processing of Earth-Abundant Cu2SnS3 Absorbers for Thin-Film Solar Cells. ACS applied materials \& interfaces 2016, 8, 11603-14.

12. Deepa, K. G.; Chandrabose, G.; Jampana, N., Nanocrystalline CuInSe 2 thin films prepared using automatic film applicator. Surfaces and Interfaces 2017, 6, 81-84.

13. Jasieniak, J.; MacDonald, B. I.; Watkins, S. E.; Mulvaney, P., Solution-processed sintered nanocrystal solar cells via layer-by-layer assembly. Nano letters 2011, 11, 2856-64.

14. Kiani, A.; Sutherland, B. R.; Kim, Y.; Ouellette, O.; Levina, L.; Walters, G.; Dinh, C.-T.; Liu, M.; Voznyy, O.; Lan, X.; Labelle, A. J.; Ip, A. H.; Proppe, A.; Ahmed, G. H.; Mohammed, O. F.; Hoogland, S.; Sargent, E. H., Single-step colloidal quantum dot films for infrared solar harvesting. Applied Physics Letters 2016, 109, 183105. 
15. Pathak, D.; Bedi, R. K.; Kaushal, A.; Kaur, D., CRYSTALLINE AgInSe2FILMS ON GLASS BY LASER ABLATION. International Journal of Modern Physics B 2010, 24, 53795385.

16. Pathak, D.; Wagner, T.; Šubrt, J.; Kupcik, J., Characterization of mechanically synthesized AgInSe2nanostructures1. Canadian Journal of Physics 2014, 92, 789-796.

17. Al-Agel, F. A.; Mahmoud, W. E., Synthesis and characterization of AIS chalcopyrite thin films for solar cell applications. Materials Letters 2012, 82, 82-84.

18. Tang, J.; Hinds, S.; Kelley, S. O.; Sargent, E. H., Synthesis of Colloidal CuGaSe2, $\mathrm{CuInSe} 2$, and $\mathrm{Cu}$ (InGa) Se2 Nanoparticles. Chemistry of Materials 2008, 20, 6906-6910.

19. Mourdikoudis, S.; Liz-Marzán, L. M., Oleylamine in Nanoparticle Synthesis. Chemistry of Materials 2013, 25, 1465-1476.

20. Huynh, W. U.; Dittmer, J. J.; Alivisatos, A. P., Hybrid nanorod-polymer solar cells. science 2002, 295, 2425-2427.

21. Pathak, D.; Wagner, T.; Adhikari, T.; Nunzi, J., Inverted ternary bulk hetrojunction hybrid photovoltaic device based on AgInSe2-polymer blend as absorber and PEDOT: PSS as hole transport layer.

22. Pathak, D.; Wagner, T.; Adhikari, T.; Nunzi, J., AgInSe 2. PCBM. P3HT inorganic organic blends for hybrid bulk heterojunction photovoltaics. Synthetic Metals 2015, 200, 102108.

23. Pathak, D.; Wagner, T.; Adhikari, T.; Nunzi, J., Photovoltaic performance of AgInSe 2conjugated polymer hybrid system bulk heterojunction solar cells. Synthetic Metals 2015, 199, 87-92.

24. Pathak, D.; Bedi, R.; Kaur, D., Growth of AgInSe2 on Si (100) substrate by thermal evaporation technique. Applied Physics A 2009, 95, 843-847.

25. Takahisa Omata, Katsuhiro Nose, and Shinya Otsuka-Yao-Matsuo, Size dependent optical band gap of ternary I-III-VI2 semiconductors nanocrystals. Journal of Applied Physics 2009,105073106

26. Yu, Y.; Fong, P. W.; Wang, S.; Surya, C., Fabrication of WS2/GaN pn Junction by Wafer-Scale WS2 Thin Film Transfer. Scientific Reports 2016, 6.

27. Park, S. Y.; Kim, B. J.; Kim, K.; Kang, M. S.; Lim, K. H.; Lee, T. I.; Myoung, J. M.; Baik, H. K.; Cho, J. H.; Kim, Y. S., Low- Temperature, Solution- Processed and Alkali Metal 
Doped $\mathrm{ZnO}$ for High- Performance Thin- Film Transistors. Advanced Materials 2012, 24, 834838.

28. Guo, Q.; Ford, G. M.; Hillhouse, H. W.; Agrawal, R., Sulfide Nanocrystal Inks for Dense $\mathrm{Cu}(\operatorname{In} 1-\mathrm{x}$ Gax $)(\mathrm{S} 1-\mathrm{y}$ Se y) 2 Absorber Films and Their Photovoltaic Performance. Nano letters 2009, 9, 3060-3065. 